\title{
Online Time Delay and Disturbance Compensation for Linear Non-Minimum Phase Systems
}

\author{
Özlem Demirtaş ${ }^{1,2}$, Mehmet Önder Efe ${ }^{3}$ \\ ${ }^{1}$ Advanced Technologies \& Systems Division, Roketsan Missiles Inc., Mamak \\ 06852, Ankara, Turkey, ozlem.demirtas@ roketsan.com.tr \\ ${ }^{2}$ Hacettepe University, Graduate School of Science and Engineering, Beytepe, \\ 06800, Ankara, Turkey, ozlem.demirtas@ hacettepe.edu.tr \\ ${ }^{3}$ Department of Computer Engineering, Hacettepe University, Beytepe Campus \\ 06800, Ankara, Turkey, onderefe@ hacettepe.edu.tr
}

\begin{abstract}
Herein, a disturbance observer is designed for linear non-minimum phase systems. A Smith Predictor is added to the system, using Recursive Least Squares (RLS), with a forgetting factor algorithm. The combination of both approaches, eliminates the restrictive feature of the classical disturbance observer, for non-minimum phase systems and removes the necessity for precise delay measurements, for the Smith Predictor structure. The results show that the proposed design procedure preserves system stability, in the presence of disturbances and time delays.
\end{abstract}

Keywords: Disturbance Observer; Recursive Least Squares; Smith Predictor

\section{Introduction}

Disturbances often occur in real systems and this has a negative effect on system stability and performance. In the past, a number of remedies have been proposed to enhance the stability and performance characteristics of feedback control systems [1].

The classical disturbance observer (DOB), estimates disturbances acting on the system, utilizing a proper inverse model and eliminates the disturbance from the control channel. However, the model inversion for non-minimum phase systems leads to unstable control loops, which require a special treatment for the right half plane (RHP) zeros [2]. This undesired situation narrows down both the simplicity and the capabilities of DOB. 
Although researchers try to make the system robust by using more complex controllers due to restrictive effect of classical DOBs, the designed controllers often achieve one control target, making the system robust against disturbances, yet sacrificing other control objectives.

In addition to external disturbances, inherent time delays are also inevitable facts observed in dynamic systems, and similar to disturbances, they disrupt the system's stability and deteriorate its operation [3] [4] [5]. Smith Predictor (SP) [6] is often used to restore the stability of such systems. In this approach, negative feedback is made from the controller output to input by using time delay model and the delay becomes a multiplier of the delay free closed loop transfer function. However, in order to design the time delay model, the actual time delay must be measured precisely, which is usually not possible in practice [7].

In this study, the aim is to create a new control design, that removes the abovementioned limitations of classical DOB and SP designs and can eliminate the negative effects of both disturbance and time delay concurrently. For this reason, both the DOB for non-minimum phase systems and the online SP design for systems with time delay are proposed to eliminate the negative effects of disturbances and time delays. As a non-minimum phase system, the altitude control of the Tower Trainer 60 unmanned aerial vehicle [8] is used and $\mathrm{H}_{\infty}$ based robust control design is preferred as the altitude controller.

In the first step, the design of a DOB for non-minimum phase systems is performed. The minimum phase approximation of the system is found by using a constrained optimization approach [9], and the inverse of the system is obtained by using this approximation. Then, input and output disturbances are fed to the system and the effect of the DOB on the system performance is observed.

In the second step, the online SP design using the Recursive Least Squares with Forgetting Factor (RLSWF) [10] method is proposed. In this phase, it is assumed that the nominal model of the system is known and the real system dynamics with delay are estimated using RLSWF. In this way the disruptive effect of delay on the system is eliminated in an online manner without the need for precise measurement of the time delay.

In the last phase, the studies done in the previous steps are combined to observe how the system stability is maintained for cases where the disturbance and delay are effective simultaneously. Then the closed loop system with uncertain elements is investigated with and without the presence of combination of proposed methods.

According to the results, it is seen that the controller alone is not capable of maintaining the stability under time delay and disturbances. On the other hand, for non-minimum phase and time delay systems, the response of the system is stable and it resembles the nominal system behavior with proposed time delay and disturbance estimation methods. 
As a result, the following features in the presented study are superior to other studies in the literature:

1) A novel, simple and reliable DOB design has been created for nonminimum phase systems.

2) By making the SP design adaptive, time delay elimination is realized without the need for actual time delay measurement. Unlike the communication disturbance observer design [11], it can work for nonminimum phase systems without the need for extra design costs.

3) By combining the new SP and DOB designs, both delay and disturbance elimination is provided. 4) Both designs can be used both separately and in combination based on the system requirements.

This paper is organized as follows. Section 2 summarizes the previous work. Section 3 gives information about the DOB structure designed for linear nonminimum phase systems and shows the optimization results. Section 4 describes how the delay is compensated by combining SP and RLSWF when it occurs in the system. Section 5 shows how system works for the case where both methods are combined. Finally, Section 6 presents the conclusions.

\section{Literature Background}

In 2004, Chen, Zhai and Fukuda [12] used the least squares method to find the minimum phase estimation of the non-minimum phase system and incorporated it to the design of the classical DOB. However, this study focuses only on the inner loop of the system with DOB structure and the disturbance estimation. Therefore, in the presence of a controller, the performance of the closed loop system is not considered.

In 2010, Kim and Son [13] also designed a DOB for non-minimum phase systems and demonstrated that the designed observer executes robustly in the presence of time delay and time varying disturbance in the input signal. This study is important as it provides delay and disturbance compensation by combining nonminimum phase DOB and classical SP designs. However, the need for actual time delay measurement and the insufficiency for time varying delays of classical SP are the limitations of the study.

In 2010, Jo, Shim and Son [14] designed a parallel filter using the $\mathrm{H}_{\infty}$ synthesis technique for non-minimum phase system. Then, using this technique, they also incorporated a robust controller into the closed loop system and showed that without the proposed DOB, the corresponding controller can only achieve one design goal. Although this design provides the applicability of the classic DOB design for non-minimum phase systems, it has two drawbacks: 
1) The input disturbance affecting the system is not injected the input of the parallel filter at runtime. For this reason, this situation leads to a small uncertainty in the design.

2) In order to calculate the parallel filter, a deep robust stability analysis is required.

In 2013, Sarıyld1z and Ohnishi [15] estimated the minimum phase equivalents by running an optimization method for the RHP zero(s) in the system and presented a study on which constraints should be considered when designing $Q$ filter for such systems. In this method, the performance of the DOB is directly dependent on the performance of the optimization method used, and an approach with a high error rate affects the DOB performance and fidelity of the simulation negatively. Therefore, the cost function and optimization method should be selected in accordance with the non-minimum phase system to be used.

In 2014, Wang and Su [16] designed a DOB for non-minimum phase, delayed systems and they presented a $Q$ filter optimization using $\mathrm{H}_{\infty}$ theory. In this study, disturbance rejection control using DOB for stable systems is investigated. However, although the study presents a design methodology considering both time delay and RHP zeros, the validity of the study only for stable systems and having a deep robust stability analysis are the limitations of the proposed method.

Observers are used quite often, not only for disturbance estimation, but also for estimating some system parameters. In 2014, Regaya et al. [17] estimated the speed in the induction machine using the sliding mode observer. Thus, they provided the control and determination of the unknown rotor speed without the need for the speed sensor. The simulation results support that the speed estimation was carried out successfully and the chattering in the controller was reduced.

The time delay problem is encountered in many different fields. In 2016, Muradore and Fiorini [18] examined the algorithms that will ensure the stability of communication between master and slave systems in dual teleoperation technologies and presented the advantages and disadvantages of these algorithms. These algorithms are based on passivity theory to guarantee the stability of teleoperation in the presence of time delay. Similarly, in 2017, Marton et al. [19] presented the modified bilateral control algorithm based on the time domain passivity concept, which guarantees system stability in the presence of time varying delays.

Although there exist DOB designs for non-minimum phase and delayed systems in the literature, an in depth stability analysis is required for designs using $\mathrm{H}_{\infty}$ theory [14] [16]. In other DOB designs for non-minimum phase systems, the delay is not included in the system [12] [15]. Even so, an accurate delay measurement is still required for SP design [13]. In this study, for non-minimum phase systems with time delays, delay compensation without precise delay information requirement is investigated and a practical optimization study for RHP zeros is conducted. 


\section{Disturbance Observer Design for Linear Non- Minimum Phase Systems}

\subsection{Classical Disturbance Observers}

The structure of classical DOB is shown in Figure 1, where $P(s)$ is the real system, $P_{n}(s)$ is the nominal model of the system, $C(s)$ is the system controller and $Q(s)$ is a low pass filter.

As shown in Figure 1, the control signal exposed to the disturbance enters the plant via the control channel. The resulting -possibly- noisy output signal passes through the inverse of the nominal system dynamics and $Q$ filter, respectively, and the estimated value of the disturbance is obtained.

The performance and stability of a closed loop system with a DOB depends tightly on the design of $Q$ filter. In [20], a design procedure, which always guarantees the closed loop stability of the $Q$ filter, is proposed. However, the use of inverse system dynamics in the design of DOB also requires some special considerations for non-minimum phase systems as the inverse of the nominal plant is unstable.

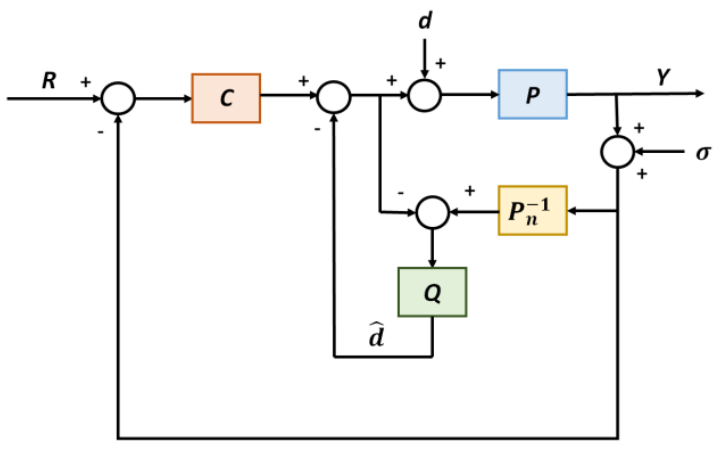

Figure 1

Classical DOB structure

\subsection{Effect of Non-Minimum Phase Systems on Stability of Classical Disturbance Observer}

If the stability condition is examined for the inner loop in which the classical DOB is located (See Figure 1), we have the following transfer functions from $r$ to $y$ in (1) and from $d$ to $y$ in (2). The measurement noise is denoted by $\sigma$ and the transfer function from $\sigma$ to $y$ is given in (3):

$P_{r y}(s)=\frac{P(s) P_{n}(s)}{P_{n}(s)+\left(P(s)-P_{n}(s)\right) Q(s)}$ 
$P_{d y}(s)=\frac{P(s) P_{n}(s)(1-Q(s))}{P_{n}(s)+\left(P(s)-P_{n}(s)\right) Q(s)}$

$P_{\sigma y}(s)=\frac{P(s) Q(s)}{P_{n}(s)+\left(P(s)-P_{n}(s)\right) Q(s)}$

If $P=P_{n}(1+\Delta)$, i.e. the uncertainty model is multiplicative, the characteristic equation of the closed loop system is as given in (4):

$P_{n}(s)+\left(P(s)-P_{n}(s)\right) Q(s)=0$

$P_{n}(s)(1+\Delta(s) Q(s))=0$

If (3) and (4) are combined, below transfer function is obtained:

$P_{\sigma y}(s)=\frac{\left(P_{n}(s)+(1+\Delta(s))\right) Q(s)}{P_{n}(s)(1+\Delta(s) Q(s))}$

In (5) $P, Q$ and $\Delta$ are can be expressed as the ratio of polynomials such that $P_{n}=N_{P_{n}} / D_{P_{n}}, Q=N_{Q} / D_{Q}$ and $\Delta=N_{\Delta} / D_{\Delta}$. Rearranging (5) with these variables yields:

$P_{\sigma y}(s)=\frac{N_{Q}\left(N_{P_{n}} D_{\Delta}+D_{P_{n}} D_{\Delta}+N_{\Delta} D_{P_{n}}\right)}{N_{P_{n}}\left(D_{\Delta} D_{Q}+N_{\Delta} N_{Q}\right)}$

In order to fulfill the internal loop stability condition, the denominator polynomial $N_{P_{n}}\left(D_{\Delta} D_{Q}+N_{\Delta} N_{Q}\right)$ specified in (6) must be Hurwitz. In this case, the nominal system must be minimum phase because $N_{P_{n}}$ stands for the numerator of the nominal system.

\subsection{Approximation of Non-Minimum Phase Systems}

In order to invert the non-minimum phase system transfer function, minimum phase equivalents of the RHP zeros in the numerator of the nominal system that make the system non-minimum phase should be found for a certain frequency range. For this purpose, the approach specified in [15] is adopted with changes in the error function.

Suppose that the non-minimum phase causal system which we want to invert contains RHP zeros and $N_{P}$ represents the polynomial consists of only RHP zeros. Also the non-causal, minimum phase transfer function that we calculated as the equivalent of $N_{P}$ at the end of optimization is $N_{\text {approx }} / D_{\text {approx }}$. In this case, the error polynomial can be defined as in (7):

$e:=N_{P}-\frac{N_{\text {approx }}}{D_{\text {approx }}}$

With this definition, the optimization problem can be cast as:

minimize

$\mathrm{E}=x_{\text {amp }}(w)|e(j w)|^{2}+x_{\text {phase }}(w)(\arg (e(j w)))^{2}$ 
subject to:

E1: $N_{\text {approx }}$ and $D_{\text {approx }}$ are Hurwitz polynomials.

$E 2: 0 \leq w \leq \min \left(\operatorname{Re}\left(z_{R H P}\right)\right)$

$x_{a m p}(w)$ and $x_{\text {phase }}(w)$ represent frequency dependent magnitude and phase weights. In the original solution, these coefficients are assumed to be constants. In the experiments, it is observed that taking these weights in the form of functions varying depending on the frequency yields more accurate results. In the minimization of the problem specified in (8), numerical solution is implemented by using the off-the-shelf interior point method and the solution is realized for frequency points up to the smallest of the RHP zeros.

\subsection{Novel Disturbance Observer Design for Non-Minimum Phase Unmanned Aerial Vehicle}

The Tower Trainer 60 autopilot design problem is used to test the new DOB method which is designed for non-minimum phase systems. The nominal transfer function of the system takes the elevator angle as input and provides altitude control. This transfer function's denominator degree is 5 and it has 3 zeros in total. One of these zeros is in the RHP and its value is approximately 12.449088 . The transfer function coefficients are shown in (9):

$$
P_{n}(s)=\frac{h(s)}{\delta_{e}(s)}=\frac{-34.16 s^{3}-144.4 s^{2}+7047 s+557.2}{s^{5}+13.18 s^{4}+95.93 s^{3}+14.61 s^{2}+31.94 s}
$$

In the first step, using (7), the following error polynomial is defined:

$$
e(s)=(s-12.449088)-\frac{N_{\text {approx }}}{D_{\text {approx }}}
$$

Solving the optimization problem in (8) with the error polynomial specified in (10), the minimum phase approximation of the non-minimum phase part of the transfer function is estimated. $x_{a m p}(w)$ and $x_{\text {phase }}(w)$ are chosen as $\exp (-10 * w)$ and 10, respectively. The estimation is realized non-causal by selecting the numerator degree 2 and denominator degree 1 of the minimum phase transfer function. In order to obtain more accurate results, minimization can be carried out for different numerator and denominator degrees by providing the condition of being non-causal. Equation (11) shows the minimum phase and non-causal transfer function obtained as a result of minimization:

$\frac{N_{\text {approx }}}{D_{\text {approx }}}=\frac{-0.183 s^{2}-5.486 s-72.63}{s+6.963}$

In Figure 2, the RHP zero polynomial ( $s$ - 12.449088), minimum phase approximation and symmetric zero polynomial $(-s-12.449088)$ is compared in Bode diagrams. In this figure, Bode diagrams display acceptable similarity up to 
the RHP zero frequency, which is used as the maximum frequency point in minimum phase approximation. This figure also shows us that the new DOB design has a bandwidth of $12 \mathrm{rad} / \mathrm{sec}$ and system responses are acceptable at frequencies below this value.

In the presence of new DOB, the robustness of the closed loop system for both input and output disturbances is studied after the minimum phase approximation of the non-minimum phase autopilot design. $\mathrm{H}_{\infty}$ synthesis method is used in the design of system controller. In this controller design type, a closed loop weighted transfer function is obtained by using the system's sensitivity and complementary sensitivity. Then, the optimal transfer function minimizing the norm of this weighted transfer function is used as system controller. Sensitivity and complementary sensitivity functions of the closed loop system are shaped using weights. For this purpose, the weighting method given in [14] is used in the control design. Values of weights are given as:

$W_{\text {Sensitivity }}(\mathrm{s})=\frac{\mathrm{s}^{2}+1.84 \mathrm{~s}+0.846}{0.001 \mathrm{~s}^{3}+1.002 \mathrm{~s}^{2}+1.84 \mathrm{~s}+1.84 \mathrm{e}-06}$

and

$W_{\text {Cosensitivity }}(\mathrm{s})=\frac{\mathrm{s}}{5} \times \frac{\mathrm{s}}{\mathrm{s}-0.001}$

The gain and phase margins of the system are calculated as $38.5 \mathrm{~dB}$ and 76.1 degrees, respectively. The reference signal, input disturbances and output disturbances are defined as $r(t)=\operatorname{step}(t), d(t)=\sin (t)$ and $\sigma(t)$ is the output measurement noise, which has uniform distribution in between $\pm 1 \mathrm{e}-3$, respectively.

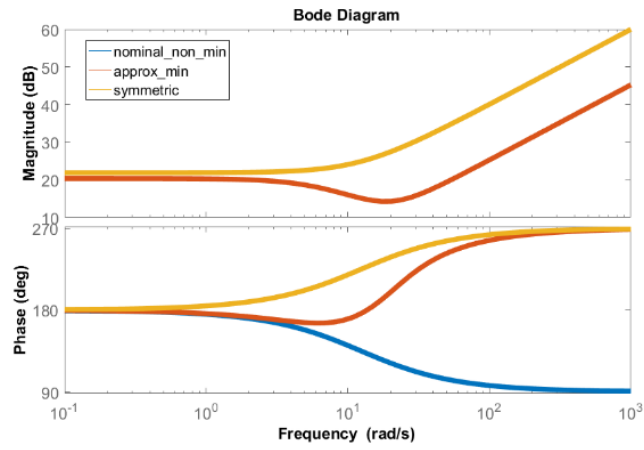

Figure 2

Bode diagrams of non-minimum phase, approximate minimum phase and symmetric polynomials. Blue, red and orange curves indicate non-minimum phase, approximate minimum phase and symmetric polynomials, respectively. 
In Figure 3 the system response obtained in the absence of DOB when input and output disturbances affect the closed loop system is shown. As can be seen from the figure, when disturbances are active, the controller becomes incapable of alleviating it and this causes oscillations in the system response.

In Figure 4 the system response obtained in the presence of controller and DOB is compared with the system responses obtained only in the presence of a controller. As can be seen from the figure, the proposed DOB design used with the controller makes the system more robust against disturbances and provides a response close to the nominal system's response.

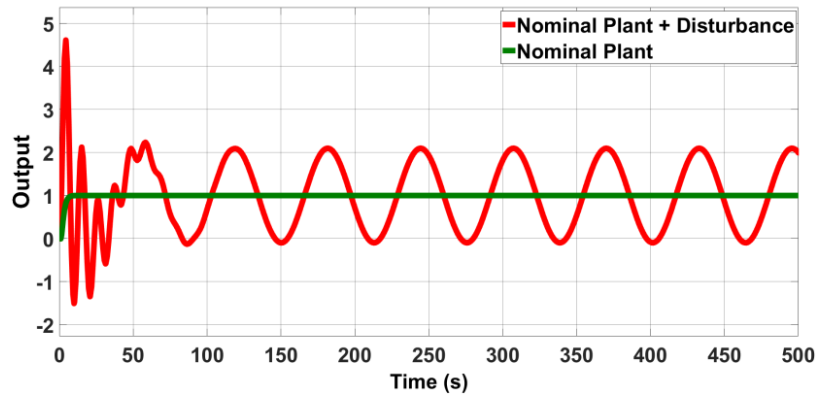

Figure 3

Closed loop responses of the system. Red and green curves show system responses in the presence and absence of both input and output disturbances, respectively.

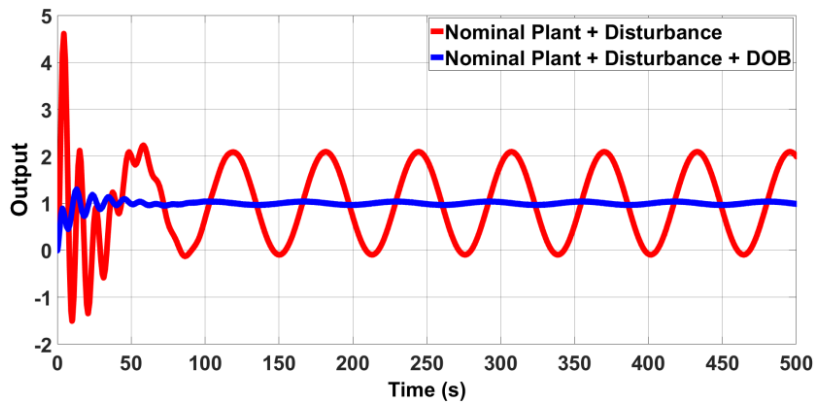

Figure 4

Closed loop responses of the system. Red and blue curves show system responses against input and output disturbances in the absence and presence of new non-minimum phase DOB, respectively. 


\section{Smith Predictor Design Using Recursive Least Squares}

\subsection{Classical Smith Predictor}

SP is a simple yet effective design that ensures a stable response from a closed loop system when time delay is available in the loop. In this design, the accurately known delay term of the system is removed perfectly from the system's characteristic equation and the negative effect of the delay on the response is eliminated. Classical SP structure is illustrated in Figure 5. As shown in the figure, the delay that adversely affects the closed loop system is compensated using the predictor transfer function $P\left(1-e^{-\tau s}\right)$.

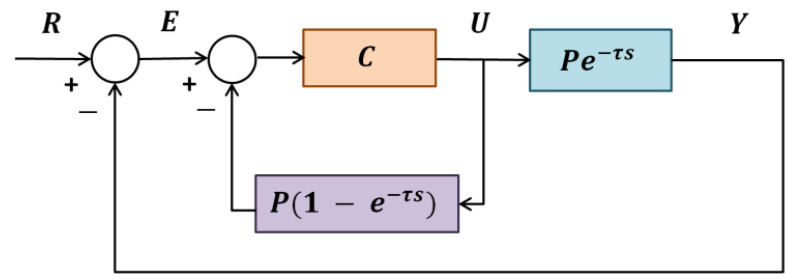

Figure 5

Classical SP structure

If the system structure is expressed using the following equations, then:

$\frac{U}{E}=\frac{C}{1+P C\left(1-e^{-\tau s}\right)}$

$\frac{Y}{R}=\frac{\frac{U}{E} \frac{Y}{U}}{1+\frac{U}{E} \frac{Y}{U}}=\frac{\frac{P C e^{-\tau s}}{1+P C\left(1-e^{-\tau s}\right)}}{1+\frac{P C e^{-\tau s}}{1+P C\left(1-e^{-\tau s}\right)}}=\frac{P C e^{-\tau s}}{1+P C}$

are obtained. Equation (13) expresses the ideal design expected in the presence of controller and SP. However, in real systems, SP efficiency depends tightly on the exact measurement of delay and correct modeling of the real system. These are rather restrictive conditions for a successful application of SP.

In Figure 6, the delayed real system $P(s) e^{-\tau s}$ is modeled and $P_{r e f}(s) e^{-\tau}$ refs is obtained. Then the SP design is studied on this new block structure. The transfer function of the new SP design is obtained as follows:

$\frac{Y}{R}=\frac{\frac{P C e^{-\tau s}}{1+P_{r e f} C\left(1-e^{-\tau_{r e f} s}\right)}}{1+\frac{P C e^{-\tau s}}{1+P_{r e f} C\left(1-e^{-\tau} r e f^{s}\right)}}$ 
$\frac{Y}{R}=\frac{P C e^{-\tau s}}{1+P_{\text {ref }} C+C\left(P e^{-\tau s}-P_{\text {refe }} e^{-\tau e f}\right)}$

As can be understood from (15), if the modeling error of the real system with delay is minimized and a sufficient level of fidelity is achieved, the ideal SP design expressed in (13) is obtained by assuming $\left(P e^{-\tau s}-P_{\text {ref }} e^{-\tau_{r e f} s}\right) \approx 0$.

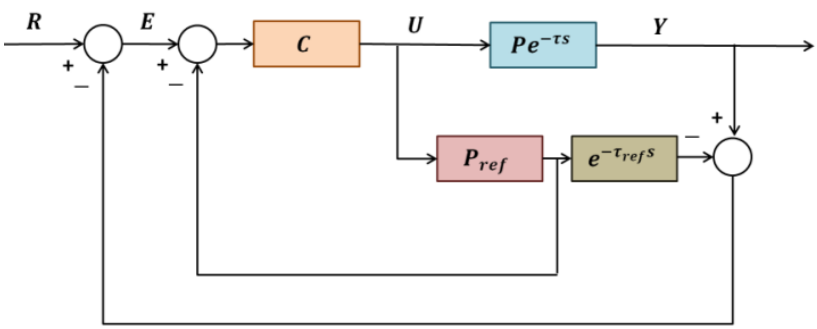

Figure 6

SP structure by modeling real system and delay

In modeling the real system with delay, classical mathematical modeling methods can be used as well as system identification techniques. In this context, RLSWF algorithm, which is a system identification method in time domain, is used in modeling the real delayed system.

\subsection{Recursive Least Squares with Forgetting Factor}

RLS algorithm is an iterative implementation of the Least Squares (LS) regression algorithm. The method allows the LS algorithm to be dynamically applied to time series obtained in real time. Algorithm is a member of Kalman filter family and exhibits an adaptive mechanism in terms of execution method. In addition, it is adjustable according to time varying input data and it has a fast convergence rate. In this respect, it clearly shows a better performance than the LS algorithm.

The basis of the LS algorithm is the identification of the linear model with unknown parameter values by minimizing the square of the difference between the real and estimated system outputs. This situation can be defined as optimizing the cost function specified in (16). In this equation, $T$ is the sampling time, $y$ is real system output, $x$ is identification input and $w$ is the vector of linear system parameters we want to obtain at the end of the identification.

$$
\epsilon(w)=\frac{1}{2} \sum_{i=1}^{m}\left(x^{T}(i T) w-y(i T)\right)^{2}
$$

If closed form solution is developed, then:

$\frac{d \epsilon}{d w}(w)=\sum_{i=1}^{m} x(i T)\left(x^{T}(i T) w-y(i T)\right)=0$ 


$$
\begin{aligned}
& \sum_{i=1}^{m}\left(x(i T) x^{T}(i T) w-x(i T) y(i T)\right)=0 \\
& w \sum_{i=1}^{m}\left(x(i T) x^{T}(i T)\right)-\sum_{i=1}^{m}(x(i T) y(i T))=0 \\
& w=\sum_{i=1}^{m}\left(x(i T) x^{T}(i T)\right)^{-1} \sum_{i=1}^{m}(x(i T) y(i T))
\end{aligned}
$$

is obtained. In the RLS algorithm, it is aimed to recursively update the equation given in (20) as the real time data are obtained. If $A(m T)=\sum_{i=1}^{m}\left(x(i T) x^{T}(i T)\right)$ and $B(m T)=\sum_{i=1}^{m}(x(i T) y(i T))$, then:

$w(m T)=A^{-1}(m T) B(m T)$

is obtained. The aim is to calculate the value of $w(m T)$ using the data we have obtained at time $(m-1) T$. In this case, the value of $w((m-1) T)$ is obtained as:

$$
w((m-1) T)=A^{-1}((m-1) T) B((m-1) T)
$$

If the values of $A(m T)$ and $B(m T)$ are also calculated using $A((m-1) T)$ and $B((m-1) T)$, then:

$$
\begin{aligned}
& A(m T)=A((m-1) T)+x(m T) x^{T}(m T) \\
& B(m T)=B((m-1) T)+x(m T) y(m T)
\end{aligned}
$$

are obtained. However, as specified in $(21), A^{-1}(m T)$ is needed to obtain the $w(m T)$ value. From the matrix inversion formula:

$A^{-1}(m T)=A^{-1}((m-1) T)-\frac{A^{-1}((m-1) T) x(m T) x^{T}(m T) A^{-1}((m-1) T)}{1+x^{T}(m T) A^{-1}((m-1) T) x(m T)}$

is obtained. If the covariance matrix $P(m T)$ and Kalman gain $L(m T)$ are shown as $A^{-1}(m T)$ and $P((m-1) T) x(m T)\left(1+x^{T}(m T) P((m-1) T) x(m T)\right)^{-1}$ respectively, $P(m T)$ can also be expressed as:

$$
P(m T)=\left(I-L(m T) x^{T}(m T)\right) P((m-1) T)
$$

In this case, to find the value of $w(m T)$ recursively, the following equations are used:

$$
\begin{aligned}
& w(m T)=P(m T) B(m T) \\
= & P(m T)(A((m-1) T) w((m-1) T)+x(m T) y(m T)) \\
= & P(m T)\left(\left(A(m T)-x(m T) x^{T}(m T)\right) w((m-1) T)+x(m T) y(m T)\right) \\
= & w((m-1) T)-P(m T) x(m T) x^{T}(m T) w((m-1) T)+P(m T) x(m T) y(m T) \\
= & w((m-1) T)+L(m T)\left(y(m T)-x^{T}(m T) w((m-1) T)\right)
\end{aligned}
$$


The algorithm structure used for RLS is similar to the one used in many recursive estimation algorithms. Differences between algorithms are mostly achieved by changing the Kalman gain. In cases where the linear system parameters are time varying, the RLS algorithm alone may not be sufficient. In this case, the forgetting factor, which is a more effective and heuristic approach, is used with RLS. This method allows more focus on recently observed data by reducing the weighting of old data points used during identification. In this case, the cost function used in the RLS algorithm, the covariance matrix $P$ and the Kalman gain $L$ are updated in the RLSWF algorithm respectively as follows:

$$
\begin{aligned}
& \epsilon(w)=\frac{1}{2} \sum_{i=1}^{m} \lambda^{m-i}\left(x^{T}(i T) w-y(i T)\right)^{2} \\
& P(m T)=\left(\frac{1}{\lambda}\right)\left(I-L(m T) x^{T}(m T)\right) P((m-1) T) \\
& L(m T)=P((m-1) T) x(m \mathrm{~T})\left(\lambda+x^{T}(m T) P((m-1) T) x(m T)\right)^{-1}
\end{aligned}
$$

The forgetting factor $(\lambda)$ value varies in between 0 and 1 , and this value provides a compromise between the stability and tracking performances of the algorithm. As this value approaches 0 , the tracking capability of the algorithm is improved, but negatively affects stability.

\subsection{Combination of Smith Predictor Solution and Recursive Least Squares with Forgetting Factor}

In the new SP design, the classical SP structure is combined with the RLSWF algorithm, aiming to eliminate the adverse effect of delay without entailing precise delay measurement. The structure used in the new SP design is shown in Figure 7. Unlike the system shown Figure 6, it is assumed that the nominal model of the system is known and only the delayed system is modeled using RLSWF. In addition, it is aimed to model only the delayed system by eliminating the necessity of modeling the delay separately.

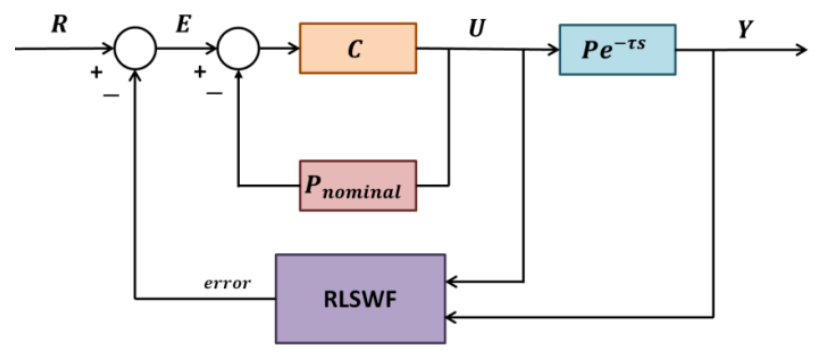

Figure 7

New SP design 
In this structure, the RLSWF algorithm uses the inputs and outputs of the delayed system for identification and yields the error value between the real and the estimated system as output. This value is then subtracted from the reference signal. Similarly, the difference between the error signal and the nominal system output is given to the system controller as input. The execution performance of the new solution is directly dependent on the performance of the RLSWF algorithm. As specified in (15), if the modeling of the delayed system is done with a sufficient level of fidelity and $\left(P e^{-\tau s}-P_{\text {ref }} e^{-\tau}\right.$ ref $\left.s\right) \approx 0$ is obtained, the ideal SP design is achieved. Therefore, if the RLSWF algorithm also performs high-fidelity identification and obtains the error value between the real and the identified delayed systems close to zero, more acceptable delay compensation is achieved by approaching the ideal SP design.

\subsection{Novel Smith Predictor Design for Unmanned Aerial Vehicle with Delay}

In order to compare the new SP design with the classical SP design, the autopilot of the Tower Trainer 60 unmanned aerial vehicle is used, in which the DOB design presented in the previous section, is also tested. In Figure 8 and Figure 9, it is observed how robust the system is against delays in the presence of a controller obtained using the $\mathrm{H}_{\infty}$ design method. Delay values are chosen as $\tau=0.1 \mathrm{~s}$ and $1 \mathrm{~s}$, respectively. The reference input is a step signal. As can be seen from the results, the controller maintains a very precise tracking under the presence of process delay. In this case, there is no need for an external structure other than the controller to reduce the deteriorating effect of the delay in the presence of the specified delay values.

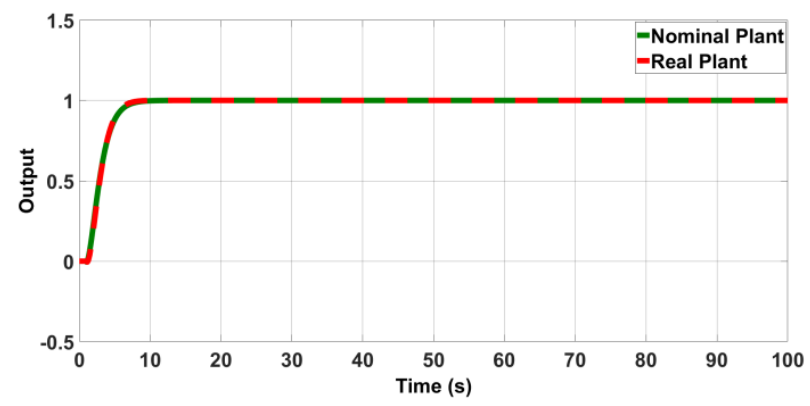

Figure 8

Closed loop responses of the system. Red and green curves show system responses in the presence and absence of $\tau=0.1 \mathrm{~s}$, respectively. 


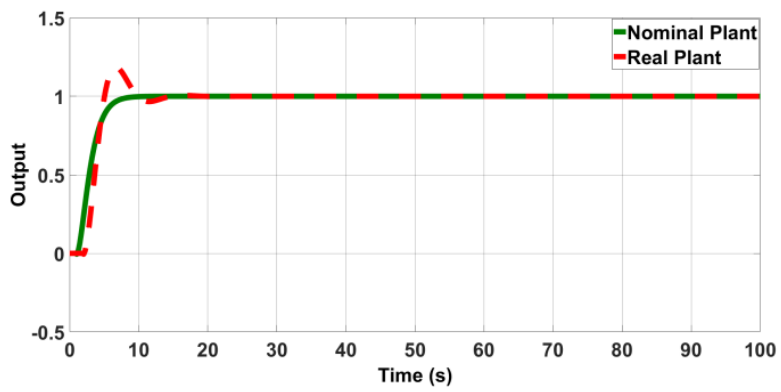

Figure 9

Closed loop responses of the system. Red and green curves show system responses in the presence and absence of $\tau=1 \mathrm{~s}$, respectively.

In Figure 10 the responses of nominal and delayed real systems are compared by choosing $\tau=10 \mathrm{~s}$. As can be seen from the figure, the system response loses its stability at high delay values and the controller cannot perform the satisfactory performance. For this reason, the novel SP design approach created using the RLSWF algorithm is added to the closed loop system and responses are examined.

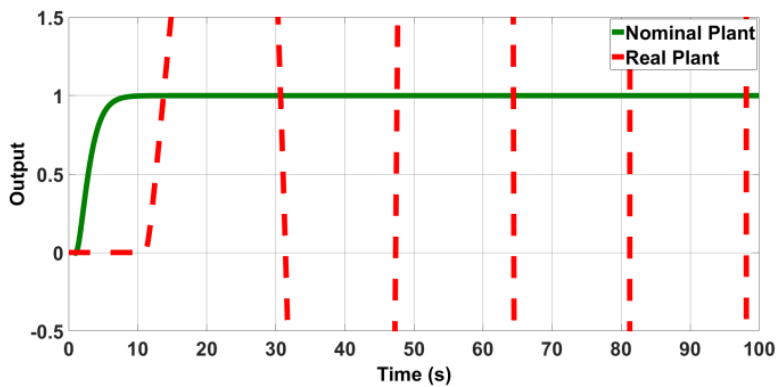

Figure 10

Closed loop responses of the system. Red and green curves show system responses in the presence and absence of $\tau=10 \mathrm{~s}$, respectively.

In Figure 11, the response of the closed loop system in which this design is used and the response of the system obtained in the presence of a controller only are compared. As can be seen from the figure, the new design ensures a stable response from the system by eliminating the deteriorating effect of the delay without the need for the actual delay information, even at high delay values. In this design, the performance of the RLSWF algorithm directly affects the performance of the SP design. Because as specified in Figure 7, RLSWF identifies the real system with delay and uses the identification error value in the closed loop system. Therefore, identification should be done at a high level of fidelity and this error rate should be kept low. Figure 12 shows both the response of linear system with $\tau=10 \mathrm{~s}$ delay and the estimated system. As can be seen from the figure, the closed loop response of the linear system estimated by RLSWF is close to the real system response. 


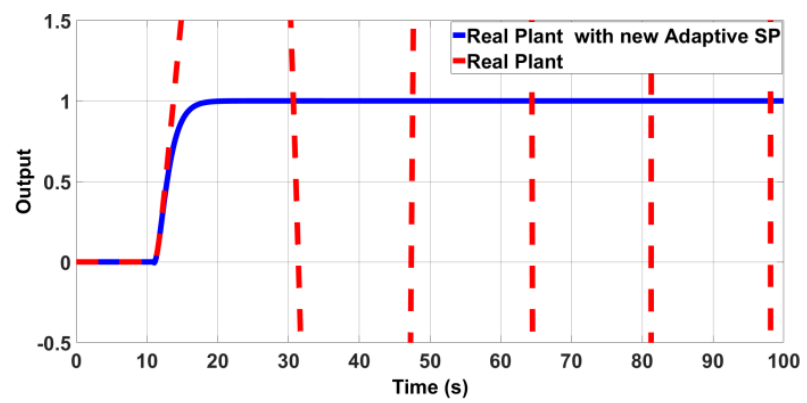

Figure 11

Closed loop responses of the system. Red and blue curves show system responses when $\tau=10 \mathrm{~s}$. The loop in the presence (blue) and absence (red) of new SP approach produces radically different responses.

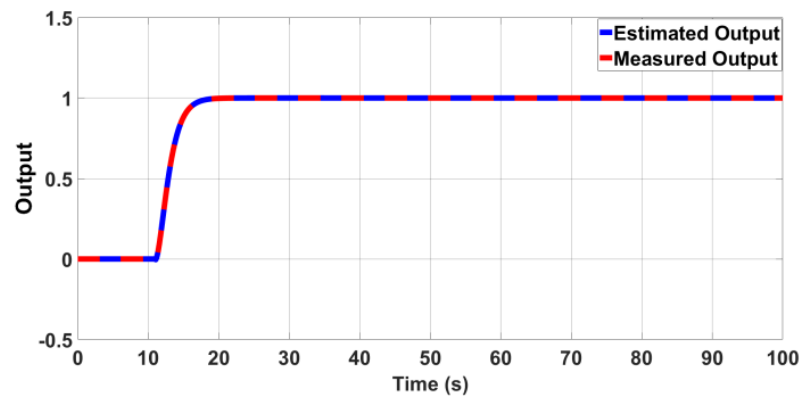

Figure 12

Identification results of the system. Blue and red curves show estimated and measured output of RLSWF, respectively.

Finally, in the Figure 13, the performances of the new SP design and classical SP design are compared in the presence of $\tau=10 \mathrm{~s}$ delay. As can be seen from the figure, while the new SP design performs better in terms of accuracy and speed, it does not require prior knowledge of precise measurement or delay.

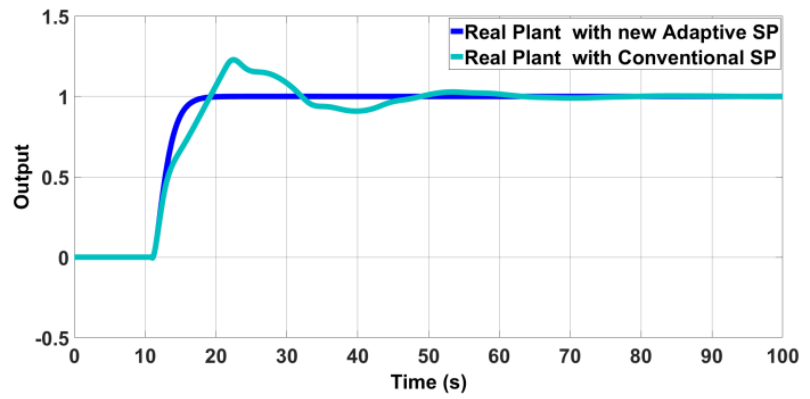

Figure 13

Responses of both classical SP and new SP design. Turquoise and blue curves indicate system responses with classical and new SP designs, respectively. 


\section{Online Disturbance and Delay Compensation Design}

In this study, the novel DOB designed for non-minimum phase systems and the SP design which is implemented without relying on the actual delay measurement are combined to create a robust system against both time delays and disturbances. The system structure formed when two designs are combined can be seen in the Figure 14. Both designs can be developed and used independently, or they can be combined as shown in the figure. However, if the two designs are used together, the nominal delay value is required for the design of the non-minimum phase DOB. Because the process of inverting the delayed non-minimum phase system is performed once and before the system is started. For this reason, although an exact measurement of the actual delay value is not needed, only for the DOB design, the presumed nominal delay value is needed. In this way, for an actual non-minimum phase system where the delay occurs, the inverse operation can be performed accurately. During the DOB design, the delay model is approximated as $e^{-\tau s} \approx$ $\frac{1}{\tau s+1}$. The unmanned aerial vehicle model, which is used for testing purposes in the proposed SP and DOB designs, is also used as a test system for the case, where the two designs are operating together.

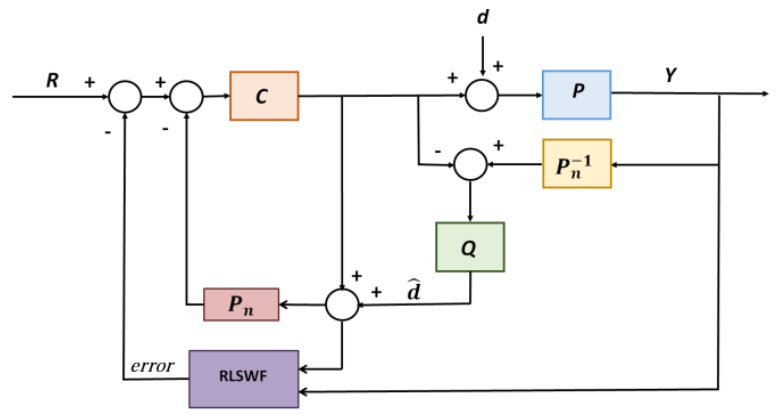

Figure 14

Online disturbance and delay compensation design

By applying the disturbances to the system with delay, system response in the presence of a controller and system responses when two designs are used together are compared. $R(t)=$ step $(t)$ is given to the system as a reference input. Disturbance is chosen as $d=\sin (t)$. Figure 15 shows the case where the delay $\tau=5 s$ and the input disturbance is given to the control signal of the system. Similarly, Figure 16 shows system response where the delay is $\tau=10 \mathrm{~s}$ and with the same disturbance signal. As can be seen from the system responses, when both designs are used in combination, the system exhibits better tracking performance under the presence of delay and disturbances. 


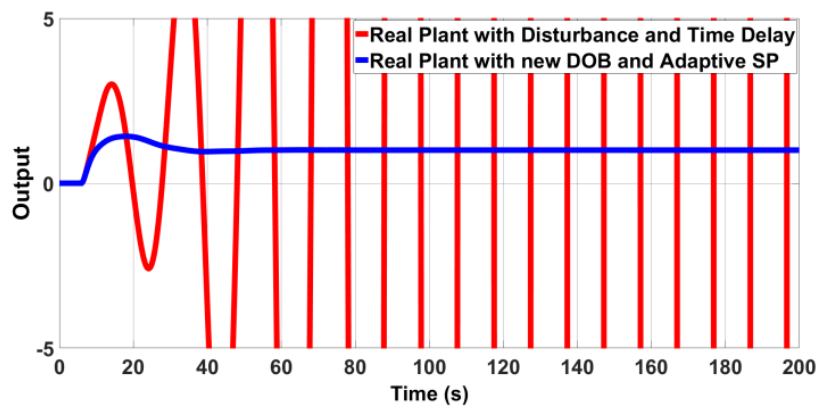

Figure 15

Closed loop responses of the system. Red and blue curves show system responses against delay $\tau=5 s$ and input disturbance in the absence and presence of new design, respectively.

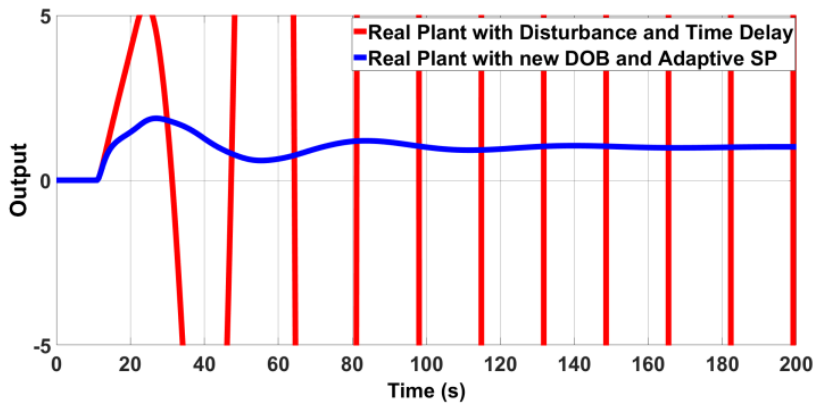

Figure 16

Closed loop responses of the system. Red and blue curves show system responses against delay $\tau=10 \mathrm{~s}$ and input disturbance in the absence and presence of new design, respectively.

\section{Conclusions}

This study combines SP and DOB approaches with an online identification mechanism. The following advantages of the proposed approach are as follows:

- The DOB design developed for non-minimum phase systems is used by updating the minimization problem specified in [15], and the requirement for the system to be the minimum phase is removed.

- In systems with delay, the classical SP design, which is proposed to be combined with the RLSWF algorithm, eliminates the need for precise measurement of the delay.

- Both designs can be used combined or separately. In this way, the system is made more robust when the controller alone is insufficient against delays and disturbances.

However, in cases where both designs are used together, the nominal value of the delay is needed, to correctly obtain the inverse of the non-minimum phase system. 
In future work, we intend to use the RLSWF algorithm, also in a DOB design, in order to eliminate the abovementioned delay value dependency.

\section{Acknowledgement}

This study is a part of the M.S. Thesis of the first author.

\section{References}

[1] A. Turnip, \& J. Panggabean: Hybrid controller design based magnetorheological damper lookup table for quarter car suspension, Int. J. Artif. Intell, Vol. 18(1), 2020, pp. 193-206

[2] H. Shim, N. H. Jo: An almost necessary and sufficient condition for robust stability of closed loop systems with disturbance observer, Automatica, Vol. 45, 2009, pp. 296-299

[3] T. Haidegger, L. Kovács, R. E. Precup, S. Preitl, B. Benyó, Z. Benyó: Cascade control for telerobotic systems serving space medicine. IFAC Proceedings Volumes, Vol. 44(1), 2011, pp. 3759-3764

[4] T. Haidegger, L. Kovács, R. E. Precup, B. Benyó, Z. Benyó, S. Preitl: Simulation and control for telerobots in space medicine, Acta Astronautica, Vol. 81(1), 2012, pp. 390-402

[5] V. M. Aparanji, U. V. Wali, R. Aparna: Multi-Layer Auto Resonance Network for Robotic Motion Control, Int. J. Artif. Intell, Vol. 18(1), 2020, pp. 19-44

[6] Y. Sun, C. Xu, H. Yu: Research of adjusted Smith predictor based on immune feedback, IEEE International Conference on Measuring Technology and Mechatronics Automation, Vol. 2, 2010, pp. 1072-1075

[7] O. Camacho, F.D. la Cruz: Smith Predictor Based-Sliding Mode Controller for Integrating Processes with Elevated Deadtime, ISA Transactions, Vol. 43, 2004, pp. 257-270

[8] I. Barkana: Classical and simple adaptive control for non-minimum phase autopilot design, J. Guidance, Control, and Dynamics, Vol. 28, No. 4, 2005, pp. 631-638

[9] A. S. Nemirovski, M. J. Todd: Interior-point methods for optimization, Acta Numerica, Vol. 17, 2008, pp. 191-234

[10] A. Vahidi, A. Stefanopoulou, H. Peng: Recursive least squares with forgetting for online estimation of vehicle mass and road grade: theory and experiments, Vehicle System Dynamics, Vol. 43, No. 1, 2005, pp. 31-55

[11] A. Krejčí, T. Popule, and M. Goubej: Closing the motion control loops via industrial ethernet network, in Proceedings of the $201415^{\text {th }}$ International Carpathian Control Conference, 2014, pp. 273-278 
[12] X. Chen, G. Zhai, T. Fukuda: An approximate inverse system for nonminimum phase systems and its application to disturbance observer, Systems \& Control Letters, Vol. 52, 2004, pp. 193-207

[13] I. H. Kim, Y. I. Son: Robust Control for Input Time Delay Systems: A Disturbance Observer Approach, SICE Annual Conference, 2010, pp. 179-183

[14] N. H. Jo, H. Shim, Y. I. Son: Disturbance Observer for Non-minimum Phase Linear Systems, International Journal of Control, Automation, and Systems, Vol. 8, 2010, pp. 994-1002

[15] E. Sarıyıldız, K. Ohnishi: A New Solution for the Robust Control Problem of Non-minimum Phase Systems using Disturbance Observer, IEEE International Conference on Mechatronics (ICM), 2013, pp. 46-51

[16] L. Wang, J. Su: Disturbance rejection control for non-minimum phase systems with optimal disturbance observer, ISA Transactions, Vol. 57, 2014, pp. 1-9

[17] C. B. Regaya, A. Zaafouri, A. Chaari: A new sliding mode speed observer of electric motor drive based on fuzzy-logic, Acta Polytechnica Hungarica, Vol. 11(3), 2014, pp. 219-232

[18] R. Muradore, P. Fiorini: A review of bilateral teleoperation algorithms, Acta Polytechnica Hungarica, Vol. 13(1), 2016, pp. 191-208

[19] L. Márton, Z. Szántó, T. Haidegger, P. Galambos, J. Kövecses: Internetbased bilateral teleoperation using a revised time-domain passivity controller, Acta Polytechnica Hungarica, Vol. 14(8), 2017, pp. 27-45

[20] H. Shim, Y. J. Joo: State space analysis of disturbance observer and a robust stability condition, IEEE Conf. on Dec. and Control, 2007, pp. 21932198 